\title{
Movement Characteristics and Heart Rate Profiles Displayed by Female University Ice Hockey Players
}

\author{
Joel Jackson \\ Faculty of Physical Education and Recreation, University of Alberta, Edmonton T6G 2H9, Canada \\ E-mail: jkjackso@ualberta.ca \\ Gary Snydmiller \\ Augustana Faculty, University of Alberta, Camrose T4V 2R3, Canada \\ E-mail: gds@ualberta.ca \\ Alex Game \\ Faculty of Physical Education and Recreation, University of Alberta, Edmonton T6G 2H9, Canada \\ E-mail: alex.game@uaberta.ca \\ Pierre Gervais \\ Faculty of Physical Education and Recreation, University of Alberta, Edmonton T6G 2H9, Canada \\ E-mail: pgervais@ualberta.ca \\ Gordon Bell (Corresponding author) \\ Faculty of Physical Education and Recreation, University of Alberta, Edmonton T6G 2H9, Canada \\ E-mail: gordon.bell@ualberta.ca
}

Received: 20-11- 2015

doi:10.7575/aiac.ijkss.v.4n.1p.43
Accepted: 22-01- 2016

Published: 31-01- 2016

\begin{abstract}
Background: Women's ice hockey is widely popular but the various movement patterns, heart rate responses and work to rest ratios during competitive games has not been adequately investigated. Objectives: This study determined the frequency and duration of movements that female players perform in ice hockey games using time-motion analysis. The intensity of the game activities were also assessed by heart rate (HR) responses and work to rest ratios (W:R). Methods: Twenty-two university female ice hockey players were filmed performing a number of movements during three regular season league games. Results: The following movement patterns were categorized in percent of time performed during the games: forward gliding on ice $(36.3 \pm 6.2 \%)$, forward skating at a moderate intensity $(31.2 \pm$ $6.2 \%)$, backward glide $(9.5 \pm 4.1 \%)$, standing $(7.1 \pm 5.9 \%)$, struggling $(6.3 \pm 2.6 \%)$, forward skating at maximal intensity $(5.3 \pm 3.3 \%)$, backwards skating at moderate intensity $(3.1 \pm 3.3 \%)$. Defense stood and glided backward more than forwards but skated less at a high or maximal intensity. Positional differences were also observed during different game play situations. The highest HR ( $\square \pm$ SD) achieved during shifts was $182 \pm 10$ and HR averaged $174 \pm 9 \mathrm{bpm}$ for the whole duration of the shifts. The shift and game $\mathrm{W}: \mathrm{R}$ ratios for all players were 1:1.6 and 1: 3.7 , respectively. Conclusions: The findings of this study indicate that female ice hockey games are played at a low to moderate intensity most of the time ( $~ 84 \%$ of the time spent) and are interspersed with brief, intermittent high intensity activities that vary according to player position and game play situation. It was also apparent that female players display markedly high HR responses during game-play indicative of a substantial cardiovascular demand in ice hockey.
\end{abstract}

Keywords: game analysis, work to rest ratios, exercise intensity

\section{Introduction}

Ice hockey is a popular sport that is played by both genders at a variety of ages and competitive levels (e.g. recreational, representative, professional), including the winter Olympic Games. Hockey is intermittent in nature and requires the players to apply a series of skillful movements on a frozen surface that vary due to the rules, format and strategy of the game (Bloomfield, Polman, O’Donoghue, \& McNaughton, 2007b; Bracko, Fellingham, Hall, Fisher, \& Cryer, 1998; Cox, Miles, Verde, \& Rhodes, 1995). Players in forward and defense positions compete in repeated shifts during three periods lasting twenty minutes each, with stop time and an intermission between the first and second period. The frequency and time spent performing the various movements by players during ice hockey games have been described and documented using video capture devices and time motion analysis techniques (TMA; Green et al., 1976; Seliger et al., 1972; Thoden, \& Jette, 1975). As well, heart rate has been recorded during ice hockey games for female (Spiering, 
Wilson, Judelson, \& Rundell, 2003) and male (Green et al., 1976) players and has been used simultaneously with TMA to further indicate the physiological demands of various activities performed during competition. Furthermore, the physiological demands of this sport also require each player to possess a combination of fitness qualities (e.g. aerobic, anaerobic, strength, power, body composition) to be successful (Geithner, Lee, \& Bracko, 2006; Ransdell \& Murray, 2011) and various correlates of skating performance have been investigated in male and female ice hockey players (Farlinger, Kruisselbrink, \& Fowles, 2007; Gilenstam, Thorsen \& Henriksson-Larsen, 2011). However, the majority of TMA research in ice hockey is more than 30 years old (Green et al., 1976; Seliger et al., 1972; Thoden \& Jette, 1975). During this time, hockey players have achieved higher levels of physical fitness due to advances in training methodology that has contributed to better performance and likely differences in certain aspects of how the game is played (Montgomery, 2006; Quinney et al., 2008). However, it is important to point out that previous TMA ice hockey research has only investigated male players and there are no published reports for female ice hockey players.

A unique aspect of ice hockey is that due to particular rules of the game, there are periods of time when one or more players of one team may be penalized due to an infraction (i.e. penalty). This results in one team having an additional player or players on the ice providing them with an advantage in trying to score (termed a "power play") while the opposing team has a distinct disadvantage since they have one or more fewer players on the ice ("penalty kill"). This game feature has not been adequately investigated in previous TMA research of ice hockey and is important since these game play situations may change the time spent and types of movements performed. Thus, a novel application of TMA research to ice hockey would be to quantify any differences in movement frequency and time spent during these different gameplay situations. Furthermore, work to rest ratios (W:R) that separate the higher intensity active components to the rest/recovery portions of the game can be calculated from TMA and have been used to further indicate the energetic demands of a sport (Bloomfield, Polman, \& O’Donoghue, 2007a; Dobson, \& Keogh, 2007). A $\mathrm{W}: \mathrm{R}$ that approaches $1: 1$ would indicate that the nature of the activity was of a high intensity whereas a ratio exhibiting more rest or recovery time (e.g. 1:5) would indicate a lower intensity for the activity. Neither TMA of game play situations nor the determination of work to rest ratios has been reported in ice hockey and this knowledge may offer further insight for coaching methodology and conditioning strategies that allow for the design of more sport specific training programs.

Since there have been no published time motion analysis of women's ice hockey that the we know of, the purpose of this study was to investigate the frequency and duration of movement patterns as well as heart rate responses and work to rest ratios during female ice hockey league games and different game play situations. It was hypothesized that there would be significant differences in the frequency and duration of movement patterns during ice hockey games between the first, second and third periods of the game, between the defense and forward positions and the penalty kill and power play game situations. It was also hypothesized that the HR responses and work to rest ratios would differ by position and game play situations.

\section{Methods}

\subsection{Participants}

A sample of convenience was used in this study. Twenty-two female hockey players (14 forwards and 8 defense) from the same Canadian Interuniversity Sport (CIS) team volunteered to be in this research study. The participants' mean age, height, and body mass was $22 \pm 3$ and $22 \pm 4$ years; $168.7 \pm 5.5$ and $171.5 \pm 6.6 \mathrm{~cm} ; 66.0 \pm 5.5$ and $72.2 \pm 5.6 \mathrm{~kg}$; for forwards and defense, respectively. Note that the defense had a greater body mass than forwards $(\mathrm{P}<0.05)$, but no other significant differences in demographic data were observed. Prior to all data collection, each participant was required to complete a Physical Activity Readiness Questionnaire (PAR-Q), as well as read and sign an informed consent which was approved by a University Research Ethics Board.

\subsection{Experimental Design}

This study was descriptive in design and used videotaping and heart rate recordings of players competing in regular season, Canadian Interuniversity Sport (CIS) games that were played against different league rivals. Three games were chosen against different opponents at different times of the regular season (October, November and December). The rationale for this was that these games occurred after the team had competed in six different exhibition games and three regular season games and was done to ensure that the level of competition and how the players performed was representative of a typical CIS ice hockey game. Home games were chosen so that the camera placement and ice arena environment would be identical for all games.

To refine the movement categories chosen for this study, the principal investigator reviewed the available research literature and created a series of movement categories with descriptions for each and distributed this to a panel of 5 individuals with combined expertise in skill analysis, coaching hockey and/or sport science research for review. A subsequent meeting was held during which video clips of CIS games were viewed and a round table discussion occurred to provide feedback and refine the movement categories and their descriptions. As a result of the panel discussion and recommendations made, each description of the different types of movements was finalized. Pilot research was then performed using actual game film and after this preliminary analysis, it was further decided that the TMA would not be separated by whether or not a player was in possession of the puck. This modification was approved by the panel of experts. As well, due to the small number in the goalie position, they were not included in any analysis. 


\subsection{Exercise Testing and Heart Rate Measurement}

Prior to the recording of the first hockey game, each player came to the exercise physiology lab where age was recorded; height was measured in $\mathrm{cm}$ using a wall mounted stadiometer (Tanita, Arlington, IL) and weight was measured in kgs using a balance beam scale (HealthoMeter, Bridgeview, IL). A maximum graded exercise test was then conducted to determine peak oxygen consumption (VO2peak) and maximum heart rate (HRmax) on a Monark cycle ergometer (Model 818E, Sweden) using a standardized protocol. Each participant was connected to a calibrated metabolic measurement system (ParvoMedics TrueOne 2400, Utah) with a Rudolph valve mouthpiece and head gear assembly (Hans Rudolph Inc., Kansas) and wore a Polar HR monitor (Polar Canada, Quebec). After a standardized 5 minute warmup at $\sim 70$ watts, the graded exercise test began at 110 watts (W) and was increased by 34 W every 2 minutes until ventilatory threshold was observed on the software's graphical display (V-Slope) after which the power output was increased by $34 \mathrm{~W}$ every minute until volitional exhaustion. Pedaling rate was maintained at $75 \mathrm{rpm}$ throughout the test. The primary criteria for peak oxygen consumption was a peak and/or plateau in oxygen consumption in combination with a respiratory exchange ratio of $>1.15$, achievement of age predicted HR maximum (within 5 bxmin-1) or a known HR maximum, and exhaustion (volitional fatigue). This protocol allowed the determination of aerobic fitness as indicted by the peak oxygen consumption as previously reported by our lab (Game, Voaklander, Syrotuik \& Bell, 2003).

Eighteen players (11 forwards and 7 defense) agreed to wear Polar® Team Sport HR monitors under their protective equipment during the games and these were pre-set to begin recording 15 minutes prior to each game and store five second HR averages to memory and were downloaded to a computer after each game. The timing of the HR monitors was synchronized with the time displayed on the video cameras. Note that the decision to wear a HR monitor was solely up to each individual player and some players anticipated that they would find wearing the HR monitor distracting during an actual game. The players were allowed to become accustomed to wearing the monitor during practises but 4 players still chose not to wear one. The primary reason cited for this was that these players felt that wearing the HR monitor strap was a distraction and believed that it may interfere with their individual performance.

\subsection{Camera Setup and Time Motion Analysis}

The video device capture and time motion analysis was based on our previous research of different sports (Forbes, Kennedy and Bell, 2013; Virr, Game, Bell \& Syrotuik, 2014). For the present study, two cameras were mounted on opposite sides of the arena, in-line with center ice at an identical height of 20 meters from the ice surface. The positioning and wide angle lens on both digital cameras (GoPro® Hero; 720p, 60fps) allowed for the entire ice surface to be captured. This also allowed for the movement analysis of all players from the same recording vantage point. The video files were stored to SD cards that were later downloaded to a computer.

As a result of the panel discussion and preliminary analysis, the proposed on-ice movement pattern categories are presented in Table 1 below.

Table 1. Movement categories and descriptions

$\begin{array}{ll}\text { Movement } & \text { Description } \\ \text { Categories } & \end{array}$

Standing or very low intensity movements

Forward start

Gliding or cruising forward

Moderate intensity forward skating

High or maximal intensity forward skating

Backward start
Stationary or displaying very little motion that would be considered expending a low amount of energy. Most often taking place away from the puck/play. Examples include: a defensive player standing on the offensive blue line, a player standing in front of the net without being engaged with an opposing player, and/or a forward anticipating a play (breakout or regroup) with little movement.

Two to three forward strides that are used to accelerate the player from a stationary or near stationary position. The movement is characterized by a strong arm swing and knee drive as well as a pronounced forward lean as the player works to overcome inertia. As the player builds speed (accelerates) the intensity increases exponentially.

No or very little skating movement. The exception being easy/low intensity strides used to maintain speed.

Stride frequency is at a moderate rate and speed is above slow, but would not be considered maximal. The player displays purposeful movements to contribute to the offensive rush or to gain defensive positioning. The players arm movements are contributing to their stride, but there is less upper body lean than with a maximal effort.

The fastest rate of stride frequency which corresponds with; forceful knee drive of the recovery leg; pronounced upper body lean; and deliberate arm movements which add to the strength of the stride. The forward start may also be included in this category if the player continued maximal intensity skating beyond the 2-3 strides of a forward start.

Two to three fast backward strides that are used to accelerate the player from a stationary or near stationary position. The movements can be characterized as an effort to overcome inertia. As the player builds speed (accelerates) the intensity increases exponentially. 
Gliding or cruising

backward

Moderate intensity

backward skating

High or maximal intensity backward skating

Struggling for position or battling for the puck
No or very little skating movement. The exception being easy/low intensity strides used to maintain speed.

Stride frequency is at a moderate rate and speed is above slow, but would not be considered maximal. The player displays purposeful movements to maintain position on an opposing player through the neutral zone.

The player is using fast, powerful pushes or fast, accelerating backwards crossovers in order to match the speed of an attacking opposing player. The backward start may also be included in this category if the player continued maximal intensity skating beyond the 2-3 strides associated with the backward start.

The player is using any number of utility movements (lateral crossovers, stops, starts) along with upper body activity to gain/maintain position on an opponent, to protect the puck, or gain possession of the puck. These activities are considered high intensity since there is forceful lower and upper body work. Examples include: corner battles, pins, battles for puck possession, and battles in front of the net.

Dartfish TeamPro 5.0 software (Fribourg, Switzerland) was used to code all ten movement categories within each of the CIS games. Each established movement category was coded to a specific pre-programmed keystroke. A trained observer watched the game while recording each player's movement through selection of the appropriate key assigned to the movement category. The software automatically recorded the number of events and the duration of each event which was then exported to Excel (Microsoft, 2010) for further analysis. The total and mean duration as well as frequency of each activity was measured. The number of shifts, shift time, game work to rest totals, intervals and ratios were determined for each player.

\subsection{Work to Rest Ratios}

Two separate work to rest ratios were created for the ice hockey players. The first took into account the time spent engaged in demanding and non-demanding movements during a shift and the other used time spent on the ice and on the bench during the periods.

1) Shift work to rest ratio: Ratio of the time spent engaged in high intensity activity to the time spent engaged in low intensity activity. High intensity activities include: forward start, moderate intensity forward skating, high or maximal intensity forward skating, backward start, moderate intensity backward skating, high or maximal intensity backward skating, and struggling for position or battling for the puck. Low intensity activities include: standing or very low intensity activity, gliding or cruising forward, gliding or cruising backward, and stoppages in play where the player remains on the ice.

2) Game work to rest ratio: Ratio of the time spent on the ice during active play to the time spent between stoppages of play on the ice and time on the bench.

\subsection{Statistics and video analyses}

Mean and standard deviations of time and frequency of each movement category were calculated for all players and averaged across all three games using Microsoft Excel 2010. The data for each player was collapsed across the three CIS hockey games and used in the following analyses.

Separate 2-way analysis of variance (ANOVA) procedures were used to compare the game characteristics, frequency of movement patterns, duration of movement patterns and HR responses between forwards and defense and between the 3 periods, with repeated measures across periods. Separate 2-way ANOVA's procedures were used to compare the frequency of movement patterns, duration of movement patterns and HR responses between both positions, and 3 gameplay situations (3). Separate 2-way ANOVA's were used to compare the work to rest ratios between forwards and defense and the 3 game-play situations. Any significant main or interaction effects were further evaluated using a Tukey's HSD multiple comparison test. All statistics were analysed using Statistica 12 (Statsoft, Oklahoma) and significance was set at $\mathrm{P}<0.05$ a priori.

Intra-observer, test-retest reliability was established utilizing the same trained observer that coded all movement patterns during one period from a selected game for ten different players on two different occasions separated by one week. The mean intra-observer intra-class coefficient (ICC) and typical error (TE) were calculated using the spreadsheet created by Hopkins (14) for the frequency and duration of all movement categories was 0.75 and 2.24 and 0.76 and 4.50, respectively. Inter-observer, test-retest reliability was also conducted between two different trained observers coding the same 10 players during the same period and game. The mean inter-observer ICC and TE for frequency and duration of all movement categories was 0.85 and 1.88 and 0.84 and 4.67, respectively.

\section{Results}

\subsection{General characteristic of the ice hockey games}

The mean number of shifts per period ( $6 \pm 1$ vs. $5 \pm 1)$, mean shift duration (48.0 \pm 8.6 vs. $43.4 \pm 5.4$ s) and total game time on ice (13:59 $\pm 0: 31$ vs. 10:16 $\pm 0: 32 ;$ min:s) were different between defense and forwards, respectively $(\mathrm{P}<0.05)$. 


\subsection{Percentage of game time spent in different movement categories}

The mean percentage of time spent in each of the different movement categories for all three periods and games combined is presented in Figure 1. The most time spent during the entire game time was during gliding or cruising and performing moderate intensity skating in the forward direction for all players $(\mathrm{P}<0.05)$. As well, the defense stood and glided or skated backward at a moderate intensity more than the forwards, while the forwards glided or skated forward at a moderate and maximal intensity more than the defense did $(\mathrm{P}<0.05)$.

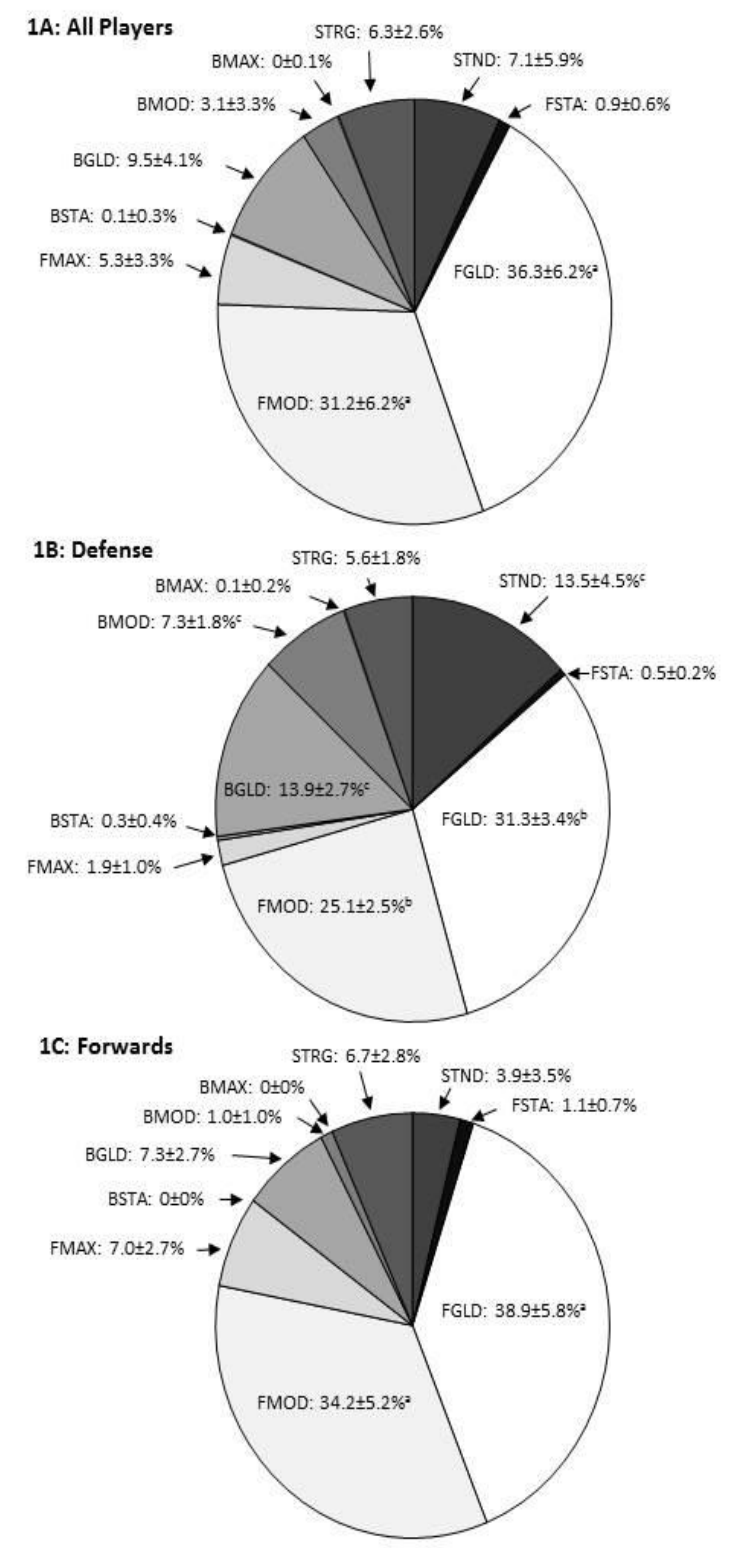

Figure 1. Percentage (\%) of time spent in each of the movement categories for all players (A) and positions (B and C) during female ice hockey games

STND=Standing; FSTA=Forward start; FGLD= forward gliding/cruising; FMOD = forward moderate intensity skating; FMAX=forward high/maximal intensity skating; BSTA=backward start; BGLD=backward gliding/cruising; $\mathrm{BMOD}=$ backward moderate intensity skating; $\mathrm{BMAX}=$ backward high/maximal skating; STRG=struggling. Values are means \pm SD.

$\mathrm{a}=$ significantly different from all other movement categories; $\mathrm{P}<0.05$.

$\mathrm{b}=$ significantly different from all other movements and forwards; $\mathrm{P}<0.05$.

$\mathrm{c}=$ significantly different from forwards; $\mathrm{P}<0.05$.

\subsection{Movement category frequencies and time}

The mean frequency and the duration of time spent in each movement category separated by player position and the 3 periods of game play for all games combined are presented in Table 2. Individuals in the defensive position stood more frequently and for longer periods of time as well as glided and skated backward at a moderate intensity more frequently and longer than forwards in all three periods $(\mathrm{P}<0.05)$. Conversely, forwards skated at a high/maximal intensity more frequently and for longer periods of time than defense did $(\mathrm{P}<0.05)$. There was also more frequent 
backward gliding for the defense in the 3rd period compared to the 1st period and this movement was performed for longer periods of time in the 2 nd and 3 rd period compared to the 1 st $(\mathrm{P}<0.05)$. It should also be noted that a statistical comparison of the backward start and high intensity backwards skating movement categories could not be performed due to a low number of these movements observed.

Table 2. Mean $( \pm \mathrm{SD})$ frequency and time spent in each of the movement categories for forwards and defense during female ice hockey games

\begin{tabular}{|c|c|c|c|c|c|c|c|c|c|}
\hline \multirow[b]{2}{*}{$\begin{array}{c}\text { Movement } \\
\text { Category }\end{array}$} & \multirow[b]{2}{*}{ Position } & \multicolumn{3}{|c|}{ Frequency (\#) } & \multicolumn{5}{|c|}{ Duration (s) } \\
\hline & & Period 1 & Period 2 & Period 3 & $\begin{array}{l}\text { Game } \\
\text { Total }\end{array}$ & Period 1 & Period 2 & Period 3 & $\begin{array}{l}\text { Game } \\
\text { Total }\end{array}$ \\
\hline \multirow{2}{*}{ STND } & $\mathrm{D}$ & $15 \pm 2^{\mathrm{a}}$ & $13 \pm 5^{\mathrm{a}}$ & $12 \pm 4^{\mathrm{a}}$ & $41 \pm 10^{\mathrm{a}}$ & $48 \pm 17^{\mathrm{a}}$ & $40 \pm 21^{\mathrm{a}}$ & $38 \pm 16^{\mathrm{a}}$ & $125 \pm 49^{\mathrm{a}}$ \\
\hline & $\mathrm{F}$ & $4 \pm 2$ & $4 \pm 3$ & $3 \pm 2$ & $11 \pm 6$ & $9 \pm 7$ & $8 \pm 8$ & $8 \pm 7$ & $25 \pm 18$ \\
\hline \multirow{2}{*}{ FSTA } & $\mathrm{D}$ & $1 \pm 1$ & $1 \pm 1$ & $1 \pm 1$ & $3 \pm 1$ & $1 \pm 1$ & $2 \pm 1$ & $1 \pm 1$ & $4 \pm 2$ \\
\hline & $\mathrm{F}$ & $1 \pm 1$ & $1 \pm 1$ & $1 \pm 1$ & $4 \pm 2$ & $2 \pm 2$ & $2 \pm 1$ & $2 \pm 1$ & $6 \pm 3$ \\
\hline \multirow{2}{*}{ FGLD } & $\mathrm{D}$ & $34 \pm 4$ & $33 \pm 7$ & $33 \pm 5$ & $100 \pm 10$ & $84 \pm 13$ & $91 \pm 16$ & $89 \pm 17$ & $264 \pm 33$ \\
\hline & $\mathrm{F}$ & $30 \pm 7$ & $28 \pm 7$ & $31 \pm 8$ & $89 \pm 13$ & $85 \pm 25$ & $77 \pm 21$ & $83 \pm 25$ & $245 \pm 50$ \\
\hline \multirow{2}{*}{ FMOD } & $\mathrm{D}$ & $34 \pm 5$ & $32 \pm 5$ & $31 \pm 4$ & $96 \pm 9$ & $71 \pm 9$ & $71 \pm 20$ & $70 \pm 9$ & $213 \pm 22$ \\
\hline & $\mathrm{F}$ & $30 \pm 6$ & $28 \pm 8$ & $30 \pm 6$ & $88 \pm 13$ & $69 \pm 16$ & $68 \pm 20$ & $72 \pm 14$ & $208 \pm 33$ \\
\hline \multirow{2}{*}{ FMAX } & $\mathrm{D}$ & $3 \pm 1^{\mathrm{a}}$ & $2 \pm 1^{a}$ & $3 \pm 1^{\mathrm{a}}$ & $9 \pm 3^{\mathrm{a}}$ & $6 \pm 4^{a}$ & $4 \pm 2^{a}$ & $6 \pm 3^{a}$ & $17 \pm 7^{\mathrm{a}}$ \\
\hline & $\mathrm{F}$ & $7 \pm 3$ & $7 \pm 2$ & $7 \pm 3$ & $21 \pm 7$ & $16 \pm 6$ & $14 \pm 6$ & $14 \pm 5$ & $43 \pm 15$ \\
\hline \multirow{2}{*}{ BSTA } & $\mathrm{D}$ & $0 \pm 1$ & $0 \pm 0$ & $0 \pm 0$ & $1 \pm 1$ & $1 \pm 1$ & $1 \pm 1$ & $1 \pm 1$ & $2 \pm 2$ \\
\hline & $\mathrm{F}$ & $0 \pm 0$ & $0 \pm 0$ & $0 \pm 0$ & $0 \pm 0$ & $0 \pm 0$ & $0 \pm 0$ & $0 \pm 0$ & $0 \pm 0$ \\
\hline \multirow{2}{*}{ BGLD } & $\mathrm{D}$ & $16 \pm 4^{a}$ & $19 \pm 5^{\mathrm{a}}$ & $21 \pm 4^{b}$ & $56 \pm 8^{a}$ & $31 \pm 10^{\mathrm{a}}$ & $43 \pm 12^{b}$ & $45 \pm 12^{b}$ & $120 \pm 24^{a}$ \\
\hline & $\mathrm{F}$ & $8 \pm 4$ & $7 \pm 3$ & $8 \pm 2$ & $24 \pm 8$ & $14 \pm 8$ & $13 \pm 6$ & $15 \pm 5$ & $43 \pm 16$ \\
\hline \multirow{2}{*}{ BMOD } & $\mathrm{D}$ & $9 \pm 3^{a}$ & $9 \pm 2^{\mathrm{a}}$ & $10 \pm 2^{\mathrm{a}}$ & $28 \pm 5^{\mathrm{a}}$ & $19 \pm 7^{a}$ & $19 \pm 4^{\mathrm{a}}$ & $24 \pm 4^{\mathrm{a}}$ & $61 \pm 9^{a}$ \\
\hline & $\mathrm{F}$ & $1 \pm 1$ & $1 \pm 1$ & $1 \pm 1$ & $4 \pm 3$ & $2 \pm 2$ & $2 \pm 2$ & $3 \pm 2$ & $6 \pm 5$ \\
\hline \multirow{2}{*}{ BMAX } & $\mathrm{D}$ & $0 \pm 0$ & $0 \pm 0$ & $0 \pm 0$ & $0 \pm 0$ & $0 \pm 0$ & $0 \pm 0$ & $0 \pm 1$ & $1 \pm 1$ \\
\hline & $\mathrm{F}$ & $0 \pm 0$ & $0 \pm 0$ & $0 \pm 0$ & $0 \pm 0$ & $0 \pm 0$ & $0 \pm 0$ & $0 \pm 0$ & $0 \pm 0$ \\
\hline \multirow{2}{*}{ STRG } & $\mathrm{D}$ & $7 \pm 2$ & $7 \pm 2$ & $7 \pm 1$ & $21 \pm 5$ & $17 \pm 7$ & $15 \pm 8$ & $13 \pm 3$ & $46 \pm 15$ \\
\hline & $\mathrm{F}$ & $7 \pm 3$ & $7 \pm 2$ & $6 \pm 3$ & $20 \pm 5$ & $15 \pm 6$ & $13 \pm 6$ & $14 \pm 7$ & $42 \pm 12$ \\
\hline
\end{tabular}

$\mathrm{D}=$ defense; $\mathrm{F}$ = forwards; STND = Standing; FSTA = Forward start; FGLD = Gliding/cruising forward; FMOD = Moderate intensity forward skating; FMAX = High/maximal intensity forward skating; BGLD = Gliding/cruising backward; BMOD = Moderate intensity backward skating; STRG = Struggling. Game Total $=$ sum of movements for all three periods.

$\mathrm{a}=$ significantly different from forwards; $\mathrm{P}<0.05$.

$\mathrm{b}=$ significantly different from forwards and from Period $1 ; \mathrm{P}<0.05$

\subsection{Game play analysis}

The mean $( \pm \mathrm{SD})$ frequency and duration of the movement categories during game play situations when players were at even strength, or when they were involved in a penalty kill or power play circumstance are found in Table 3 . Defense stood more frequently and for longer periods of time during even strength play compared to the forwards in all game play situations $(\mathrm{P}<0.05)$. Defense also stood more during the penalty kill compared to forwards during the power play or even strength play and stood less during power plays compared to the penalty kill or even strength situations $(\mathrm{P}<0.05)$. All players preformed longer maximum forward starts during the power plays $(\mathrm{P}<0.05)$. Further, all players performed forward moderate intensity skating for the longest time during power plays $(\mathrm{p}>0.05)$ and significantly less frequently during the penalty kill than both other game play situations and for the least amount of time $(\mathrm{p}<0.05)$. Maximum forward skating was performed for longer periods of time for the forwards during the penalty kill compared to the defensemen during even strength and penalty kill situations $(\mathrm{P}<0.05)$. Defense glided backward significantly more frequently and longer than forwards during all game play situations and more frequently than forwards during the power play $(\mathrm{P}<0.05)$. Defense also skated backwards at a moderate intensity more often and longer than the forwards in all three gameplay situations and longer during even strength play compared to the forwards during the power play $(\mathrm{P}<0.05)$. Finally, struggling was greatest during the penalty kill compared to when the team was at even strength or during the power play. Forwards struggled significantly more and for longer times during all gameplay situations compared to the defense during power plays. However, the defense struggled more and longer during the penalty kill than even strength and power play and were different from the forwards during the power play $(\mathrm{P}<0.05)$. 
Table 3. Mean $( \pm \mathrm{SD})$ frequency and time spent for forwards and defense during different game-play situations in individual regular season, CIS female ice hockey games

\begin{tabular}{|c|c|c|c|c|c|c|c|}
\hline & & \multicolumn{3}{|c|}{ Frequency (\#) } & \multicolumn{3}{|c|}{ Duration (s) } \\
\hline & & Even & Penalty & Power & Even & & \\
\hline $\mathrm{MC}$ & Position & Strength & Kill & Play & Strength & Penalty Kill & Power Play \\
\hline \multirow{2}{*}{ STND } & $\mathrm{D}$ & $3 \pm 0^{\mathrm{a}}$ & $2 \pm 1^{b, c}$ & $1 \pm 1^{\mathrm{e}, \mathrm{f}}$ & $8.6 \pm 2.3^{\mathrm{a}}$ & $6.6 \pm 2.0^{\mathrm{b}, \mathrm{c}}$ & $2.6 \pm 2.5^{\mathrm{e}, \mathrm{f}}$ \\
\hline & $\mathrm{F}$ & $1 \pm 0$ & $1 \pm 1$ & $1 \pm 1$ & $2.0 \pm 1.2$ & $3.8 \pm 3.1$ & $2.0 \pm 1.6$ \\
\hline \multirow{2}{*}{ FSTA } & $\mathrm{D}$ & $0 \pm 0$ & $1 \pm 0$ & $2 \pm 2$ & $0.3 \pm 0.1$ & $0.7 \pm 0.4$ & $2.1 \pm 2.4^{\mathrm{e}}$ \\
\hline & $\mathrm{F}$ & $0 \pm 0$ & $1 \pm 1$ & $1 \pm 1$ & $0.6 \pm 0.4$ & $1.8 \pm 1.7$ & $1.2 \pm 1.4^{\mathrm{e}}$ \\
\hline \multirow{2}{*}{ FGLD } & $\mathrm{D}$ & $7 \pm 0$ & $8 \pm 1$ & $9 \pm 4$ & $18.6 \pm 1.4$ & $21.7 \pm 2.7$ & $23.6 \pm 5.0$ \\
\hline & $\mathrm{F}$ & $9 \pm 1$ & $10 \pm 2$ & $9 \pm 1$ & $23.6 \pm 3.6$ & $23.5 \pm 5.8$ & $23.9 \pm 5.3$ \\
\hline \multirow{2}{*}{ FMOD } & $\mathrm{D}$ & $7 \pm 1^{f}$ & $6 \pm 0$ & $8 \pm 2^{f}$ & $15.0 \pm 1.0^{f}$ & $13.1 \pm 2.5$ & $18.1 \pm 3.5^{\mathrm{e}, \mathrm{f}}$ \\
\hline & $\mathrm{F}$ & $9 \pm 1^{\mathrm{f}}$ & $7 \pm 1$ & $9 \pm 1^{\mathrm{f}}$ & $20.2 \pm 2.6^{\mathrm{f}}$ & $14.7 \pm 3.0$ & $21.6 \pm 3.9^{\mathrm{e}, \mathrm{f}}$ \\
\hline \multirow{2}{*}{ FMAX } & $\mathrm{D}$ & $1 \pm 0$ & $1 \pm 0$ & $2 \pm 2$ & $1.2 \pm 0.4^{\mathrm{d}}$ & $1.3 \pm 0.9^{\mathrm{d}}$ & $3.0 \pm 2.7$ \\
\hline & $\mathrm{F}$ & $2 \pm 1$ & $2 \pm 2$ & $2 \pm 2$ & $4.5 \pm 1.5$ & $5.6 \pm 4.1$ & $3.7 \pm 3.1$ \\
\hline \multirow{2}{*}{ BSTA } & $\mathrm{D}$ & $0 \pm 0$ & $0 \pm 0$ & $0 \pm 0$ & $0.2 \pm 0.2$ & $0.0 \pm 0.0$ & $0.0 \pm 0.1$ \\
\hline & $\mathrm{F}$ & $0 \pm 0$ & $0 \pm 0$ & $0 \pm 0$ & $0.0 \pm 0.0$ & $0.0 \pm 0.0$ & $0.0 \pm 0.0$ \\
\hline \multirow{2}{*}{ BGLD } & $\mathrm{D}$ & $4 \pm 1^{c}$ & $3 \pm 1$ & $3 \pm 2$ & $8.4 \pm 1.5^{\mathrm{a}}$ & $7.4 \pm 0.7^{\mathrm{a}}$ & $7.6 \pm 5.2^{\mathrm{a}}$ \\
\hline & $\mathrm{F}$ & $2 \pm 1$ & $3 \pm 1$ & $2 \pm 1$ & $4.2 \pm 1.2$ & $5.9 \pm 1.8$ & $3.4 \pm 1.9$ \\
\hline \multirow{2}{*}{ BMOD } & $\mathrm{D}$ & $2 \pm 0^{\mathrm{a}}$ & $1 \pm 0^{\mathrm{a}, \mathrm{e}}$ & $1 \pm 1^{\mathrm{a}, \mathrm{e}}$ & $4.0 \pm 0.6^{\mathrm{a}, \mathrm{c}}$ & $3.2 \pm 0.6$ & $3.4 \pm 2.2$ \\
\hline & $\mathrm{F}$ & $0 \pm 0$ & $0 \pm 0$ & $0 \pm 0$ & $2.4 \pm 0.7$ & $3.2 \pm 0.9$ & $2.0 \pm 1.1$ \\
\hline \multirow{2}{*}{ BMAX } & $\mathrm{D}$ & $0 \pm 0$ & $0 \pm 0$ & $0 \pm 0$ & $0.1 \pm 0.1$ & $0.0 \pm 0.1$ & $0.0 \pm 0.1$ \\
\hline & $\mathrm{F}$ & $0 \pm 0$ & $0 \pm 0$ & $0 \pm 0$ & $0.0 \pm 0.0$ & $0.0 \pm 0.0$ & $0.0 \pm 0.0$ \\
\hline \multirow{2}{*}{ STRG } & $\mathrm{D}$ & $2 \pm 0$ & $3 \pm 0^{\mathrm{c}, \mathrm{e}}$ & $0 \pm 1^{\mathrm{a}, \mathrm{f}}$ & $3.2 \pm 1.0^{f}$ & $5.7 \pm 1.6^{\mathrm{e}}$ & $0.7 \pm 1.0^{\mathrm{a}, \mathrm{e}, \mathrm{f}}$ \\
\hline & $\mathrm{F}$ & $2 \pm 0$ & $2 \pm 1$ & $2 \pm 1$ & $4.3 \pm 1.3$ & $3.9 \pm 2.2$ & $3.8 \pm 2.9^{\mathrm{e}, \mathrm{f}}$ \\
\hline
\end{tabular}

$\mathrm{MC}=$ Movement category; D = Defense; F = Forwards; STND = Standing; FSTA = Forward start; FGLD = Gliding/cruising forward; FMOD = Moderate intensity forward skating; FMAX = High/maximal intensity forward skating; BGLD = Gliding/cruising backward; BMOD = Moderate intensity backward skating; STRG = Struggling.

Game Total $=$ sum of movements for all three periods;

$\mathrm{a}=$ significantly different from forwards in all game play situations; $\mathrm{P}<0.05$.

$\mathrm{b}=$ significantly different from forwards during even strength play; $\mathrm{P}<0.05$.

$\mathrm{c}=$ significantly different from forwards during power play; $\mathrm{P}<0.05$.

$\mathrm{d}=$ significantly different from forwards during penalty kill; $\mathrm{P}<0.05$.

$\mathrm{e}=$ significantly different even strength play; $\mathrm{P}<0.05$.

$\mathrm{f}=$ significantly different during penalty kill; $\mathrm{P}<0.05$.

\subsection{Heart rate analysis}

All players regardless of position had elevated peak and mean heart rate responses while playing (shifts) compared to the recovery heart rates between the shifts in play when sitting on the bench and the heart rates recorded during the intermissions spent in the dressing room between periods of play (Table 4). The lowest heart rate and the mean heart rate recorded during the first intermission recovery period was different from the second intermission for the forwards but there were no differences observed for the defense. Also, all players displayed significantly lower heart rates during the recovery time between shifts in the first period compared to the second period, but not the third (Figure 2).

Table 4. Mean $( \pm \mathrm{SD})$ heart rate measures for all players during female ice hockey games

\begin{tabular}{lccc}
\hline HR Measurement & Defense & Forwards & All Players \\
\hline Peak Shift HR & $184 \pm 12^{\mathrm{a}}$ & $180 \pm 7^{\mathrm{a}}$ & $182 \pm 10^{\mathrm{a}}$ \\
Mean Shift HR & $176 \pm 11^{\mathrm{a}}$ & $173 \pm 7^{\mathrm{a}}$ & $174 \pm 9^{\mathrm{a}}$ \\
Low HR Between Shifts & $129 \pm 14$ & $132 \pm 10$ & $131 \pm 12$ \\
Mean HR Between Shifts & $148 \pm 14$ & $148 \pm 9$ & $148 \pm 11$ \\
INT 1 Low HR & $103 \pm 7$ & $99 \pm 8^{\mathrm{b}}$ & $101 \pm 8$ \\
INT 1 Mean HR & $116 \pm 9$ & $112 \pm 7^{\mathrm{b}}$ & $114 \pm 8$ \\
INT 2 Low HR & $101 \pm 9$ & $108 \pm 10$ & $105 \pm 10$ \\
INT 2 Mean HR & $117 \pm 10$ & $123 \pm 8$ & $121 \pm 9$ \\
\hline
\end{tabular}

$\mathrm{a}=$ significantly different from all recovery heart rates during shifts and during intermissions.

$\mathrm{b}=$ significantly different from peak and mean shift HR and the second intermission HR. 


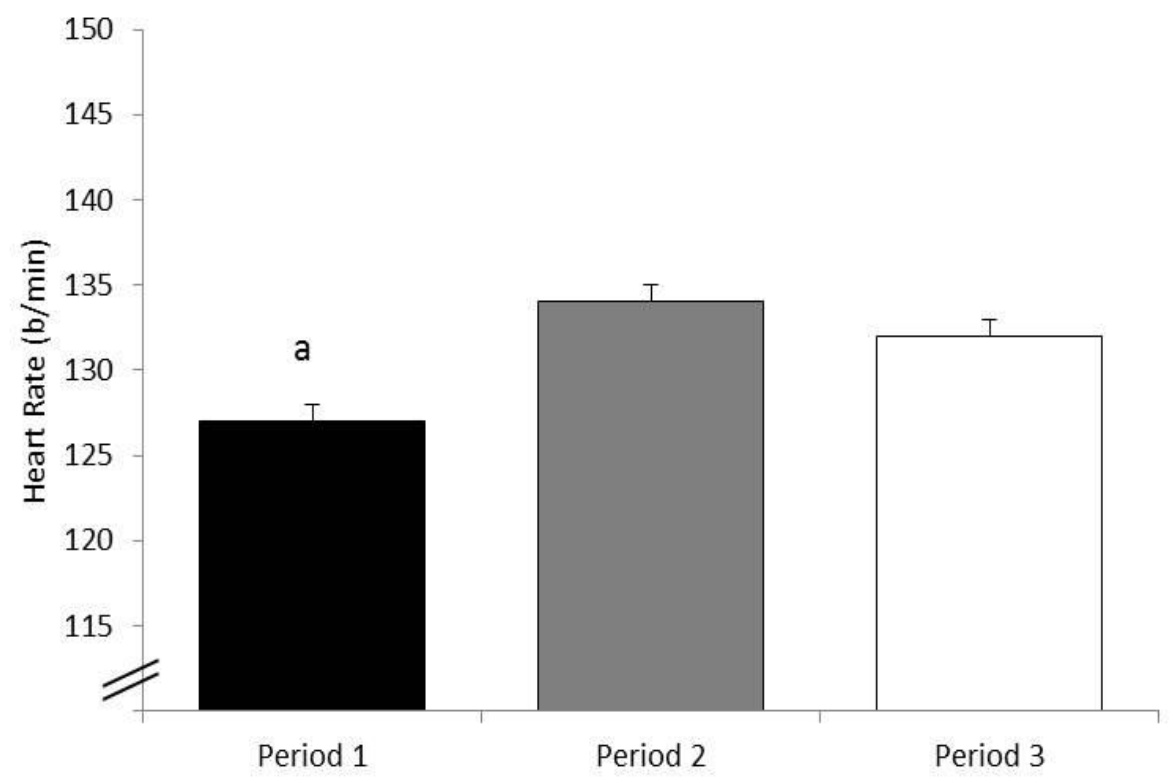

Figure 2. Lowest mean $( \pm \mathrm{SD})$ recovery heart rate measurements during the three periods for all players during female $\mathrm{a}=$ significantly different from period 2 . ice hockey games

\subsection{Work to Rest Ratios}

Table 5. Mean $( \pm \mathrm{SD})$ work to rest ratios $(\mathrm{W}: \mathrm{R})$ for all players during different game-play situation for female ice hockey games

\begin{tabular}{cclll}
\hline & Position & Even Strength & Penalty Kill & Power Play \\
\hline \multirow{3}{*}{ Shift W:R } & D & 1 to $2.0 \pm 0.2$ & 1 to $2.2 \pm 0.5$ & 1 to $2.0 \pm 0.4$ \\
& F & 1 to $1.3 \pm 0.5$ & 1 to $1.5 \pm 0.7$ & 1 to $1.2 \pm 0.5$ \\
& AP & 1 to $1.6 \pm 0.5$ & 1 to $1.8 \pm 0.7 \mathrm{a}$ & 1 to $1.5 \pm 0.6$ \\
Game W:R & D & 1 to $3.1 \pm 0.5$ & 1 to $3.7 \pm 2.8$ & 1 to $1.3 \pm 0.7$ \\
& F & 1 to $4.1 \pm 1.1$ & 1 to $6.1 \pm 6.5$ & 1 to $2.2 \pm 3.1$ \\
& AP & 1 to $3.7 \pm 1.0$ & 1 to $5.1 \pm 5.3$ & 1 to $1.8 \pm 2.4$ \\
\hline
\end{tabular}

$\mathrm{D}=$ defense $; \mathrm{F}=$ forwards; $\mathrm{AP}=$ all players.

$\mathrm{a}=$ significantly different from power play, $\mathrm{P}<0.05$.

\section{Discussion}

The popularity of female ice hockey has increased and has experienced a rise in participation of $59 \%$ from 2002 to 2013 (Hockey Canada, 2014). However, the sport science research quantifying the movement characteristics and physiological demands has been not been reported in female hockey (Geithner, Lee \& Bracko, 2006; Spiering et al., 2003). This type of information can be gleaned from time motion analysis research (Barris \& Button, 2008) and has much value in understanding the demands of any sport as well as providing a scientific basis to the design of more effective training programs, sport specific assessments, developing various coaching strategies and document any disparities in the demands of different player positions or game play situations unique to the sport (Dobson \& Keogh, 2007; Taylor, 2003). Thus, it was deemed important to investigate the frequency of occurrence and time spent performing different movement patterns using time motion analysis typical of female ice hockey games. A hierarchy of movement patterns was revealed and there were significant differences in frequency and duration of several movement activities between the defense and forward positions. There were also significant differences in the frequency and duration of certain movements during different game-play situations (i.e. even strength vs. power play vs. penalty kill). Heart rate was significantly elevated during game activities and reached near maximum levels in short periods of time during a shift made up of various activities of different intensities. Work to rest ratios supported that the sport of women's ice hockey was intermittent in nature, with the majority of time spent in low to moderate activities interspersed with high intensity, short duration skating that varied depending on the game play situation.

The findings of this study revealed that all players, regardless of position spent the majority of the game time in the forward glide $(36.3 \%)$ and moderate intensity forward skating $(31.2 \%)$ movement categories. This was similar to that reported by Bracko et al. (1998) for professional male hockey players that spent $39.0 \%$ of their total ice time in a two foot skating glide; $16.2 \%$ in a cruise stride; and, $10.0 \%$ in a medium intensity stride. This indicated that players devote the majority of their on-ice time engaged in low to moderate intensity movement categories while moving forward regardless of position. As was expected, the present study showed that defense stood and moved backwards more 
frequently but moved forward less frequently compared to forwards which has also been shown in other research (Lafontaine, Lamontagne \& Lockwood, 1998). This can be explained by the contrast in positional demands, where defense are required to skate backwards more frequently (staying between the attacking opposition and their net) and often must remain stationary in certain areas of the ice (e.g. front of the net, blue line) more often than the forwards.

Dillman, Stockholm, and Greer (1984) and Green et al. (1976) found a higher mean skating velocity for forwards compared to defense in male hockey players, but differences in forward sprinting frequency and duration between positions has not been reported for female players. In the present study, the forwards skated at a high and/or maximal intensity more frequently and for longer periods of time than players in the defense position throughout a whole game. This may be partially explained by the fact that the forwards are required to move the puck up the ice for scoring opportunities and may also be related to the aggressive fore-checking nature often required by the forward position in ice hockey games. It was also observed that the defense glided backward more frequently in the 3rd period compared to the 1st and for longer periods of time in the 2nd and 3rd period. This may have been because players in this position adopted a more defensive style of play at this point during the game given that the present team was either tied with the opposition or had more goals at the beginning of the 3rd period. It is difficult to compare these differences in the present study with other research since little TMA research has been conducted in female ice hockey players.

One aspect of ice hockey games that may change how frequent and for how long players spend performing different onice activities is during game play situations when a penalty has been assessed and one or more players are removed from the play (penalty kill) resulting in one team having a player advantage (power play) over the other. The strategy and positioning when defending during a penalty and when on the offensive with an extra player is different than when there has been no penalty assessed to either team (even strength) in ice hockey. In a penalty kill situation, a team is trying to keep their opposition in the outside perimeters of the zone created in front of the net to eliminate passing and shooting while the team with the player advantage attempt to move the puck around the defensive perimeter rapidly to create a "good" scoring chance. The present study showed that the defense stood less during the power play when a greater movement of the puck is required and often conceded by the opposing team due to the player advantage. All players skated forward at a moderate intensity less frequently during the penalty kill when establishing position is most important for defending. Defense also stood and skated backward more often and for longer periods of time compared to forwards in all game play situations. Struggling for position or to gain possession of the puck was greatest during the penalty kill game situation for all players likely because this would be a priority for effective defending. However, the defense struggled less than forwards during all gameplay situations but more often during the power play, likely due to attempting to keep the puck in the offensive zone. Lafontaine et al. (1998) reported some differences in skill frequencies between game-play situations in men's ice hockey, but this information was limited. Thus, the movement pattern profile during different game-play situations in ice hockey does vary significantly as dictated by the strategy employed for these situations.

\subsection{Heart Rate Reponses}

Heart rate monitoring using portable monitors provides continuous feedback of the physiological state of each player that can be attained during actual sport performance (Forbes, Kennedy \& Bell, 2013, Spiering et al., 2003; Stanula \& Roczniok, 2014; Virr, Game, Bell \& Syrotuik, 2014). The heart rate data revealed that there was a high cardiovascular demand placed on the female ice hockey players during games, with average peak and mean shift heart rates measured reaching $96 \%$ and $92 \%$ of maximum heart rate, respectively. These high heart rate responses are quite remarkable given the short time a player was on the ice during a shift (range of $32-54 \mathrm{~s}$ ) and the intermittent nature of low to high activities performed within the brief time of a single shift. Our data was supported by Spiering and colleagues (2003) whom also reported on-ice mean heart rates of $90 \%$ of maximum in female hockey players while Bell et al. (2011) and Green et al. (1976) reported heart rates been 94 to $98 \%$ and 87 to $92 \%$ of maximum in male hockey players, respectively. There were no significant differences in peak and mean HR during shifts between forwards and defense despite the expectation that forwards would have a greater cardiovascular response due to the anticipated higher intensity activities of playing this position. The present findings were contrary to the difference in peak and mean shift heart rates for defense and forwards in males reported by Bell et al. (2011) and could be due to a difference in shift time and the frequency and duration of activities performed by male and female players. The reason for these high heart rate responses during short, intermittent activities performed by hockey players could be due to several factors including an increase in sympathetic neural activity, elevated catecholamine concentrations, increased chemoreceptor stimulation from circulating ions, increased afferent neural activity from mechano-receptors and/or an increase in aerobic energy demand. Further research would be necessary to determine this.

Few studies have published heart rate measured in between shifts or periods in ice hockey as an indicator of recovery. In the present study, heart rate recovered to between 70 and $80 \%$ of maximum between shifts and between 56 and $64 \%$ of maximum in between periods. The recovery heart rates between shifts were significantly lower in the first period compared to the second period but not during the third period for all players. The forwards also displayed significantly higher heart rates during the second intermission compared to the first. Certainly differences in player fatigue and the associated metabolic by-products, thermal stress, possible dehydration during the game and cardiorespiratory fitness level (Linseman, Palmer, Sprenger \& Spriet, 2014; Lythe \& Kilding, 2011; Matthew \& Delextrat, 2009; McInnes, Carlson, Jones \& McKenna, 1995) may have influenced recovery heart rate to a greater extent during the second intermission for the forwards. However, despite similar elevated heart rate during activities throughout shifts, the 
defense showed similar decreases in recovery heart rate during both intermissions. Another factor that could influence recovery heart rate is competition stress. Fernandez-Fernandez et al. (2014) reported that elite female tennis players displayed significantly higher mean HR during matches that they were losing compared to those that they were winning. In this study, the team was not losing during the third period in two games and was tied in the other game where the opposition scoring the winning goal with three minutes left in regulation time. Finally, game play situations were also shown to influence recovery heart rate with higher heart rates observed during the power play compared to even strength play. The reason for this may be that there were less stationary and low intensity activities observed during power plays compared to even strength play. However, no previous research has reported heart rate responses during different game-play situations in female ice hockey.

\subsection{Work to Rest Ratios}

Work to rest ratios have been reported in TMA studies on team sports especially those that have repeated or intermittent activities and are used to further indicate the demands of a competition or certain parts of the competition by specifying how much time is spent performing activities of moderate-high intensity (work) compared to how much time is spent in low intensity activities and in recovery and depending on the ratio, it may reflect the energy demands of playing a sport (Bloomfield et al., 2007a; Dobson \& Keogh, 2007; O’Donoghue, 2008; Rudkin \& O'Donoghue, 2008). The shift work (high intensity) to rest (low intensity) ratio refers to on-ice activities; where a work to rest ratio that approaches 1:1 indicates frequent bursts of high intensity activity separated by short rest periods, and a lower work to rest ratio (e.g. 1:5) indicates that bursts of high intensity movement are separated by longer rest periods. The game work to rest ratio refers to the time spent on-ice versus and the time spent sitting on the bench. A work to rest ratio approaching 1:1 would be an indication of a short amount of bench time between shifts, whereas a lower ratio (e.g 1:5) indicates an extended time on the bench between shifts.

The average shift and game work to rest ratio in all players during even strength play was 1:1.6 and 1:3.7, respectively. Bloomfield et al. (2007a) observed a work to rest ratio of 1:1.6 that distinguished purposeful movements from nonpurposeful movements in elite male soccer players. Although this ratio was the same as the shift ratio in the present study it is important to point out that the purposeful movements reported by Bloomfield et al. (2007a) included various low intensity activities. In this study, the high intensity movements for the shift work to rest ratio also included moderate intensity backward and forward skating that could also be considered purposeful, however many instances of these movements throughout the games were somewhat low in intensity. The division of purposeful and nonpurposeful movements may lead to an overestimation of the contribution of high intensity movements in ice hockey and other sports (Bloomfield et al., 2007a). It is also difficult to directly compare work to rest ratios in other sports such as soccer where players remain in play for the majority of the game versus a sport like ice hockey where players consistently substitute off and have time to recover.

The results of this study also revealed that the shift work to rest ratio for penalty kills was lower than during power plays, but not even strength play. It was expected that both penalty kills and power plays would have lower shift W:R's compared to even strength play indicating a lower intensity compared to even strength play. This contradictory finding may have been due to a more passive style of penalty kill adopted by the team in the current study, characterized by an increase in stationary/low intensity activity with more focus on position in the defensive zone. It may also be because the current team struggled to gain possession of the offensive zone during power plays which resulted in continually trying to regroup and break the puck out of their own zone; a scenario that would be characterized by a significant amount of high intensity forward skating.

\subsection{Limitations}

The results of this study are based on a convenient sample of female ice hockey players belonging to the same team that competed during a regular CIS season. Three different games against different opponents at various times of the season were analysed in the present study. The scores for all games were within two goals, with two wins and one loss by the present team and the number of shots for and against was $22 \pm 4$ and $23 \pm 2$, respectively. These findings indicate that the observed games were similar in play and competitive in nature. Not all players agreed to wear the heart rate monitors during game play as they personally felt it was a distraction even though there was no heart rate feedback provided to participants during the game and the monitor chest straps were not restricting in anyway. The time-motion analysis methods used in this study required an observer to subjectively categorize the movement patterns of female hockey players and although this method has been widely published, the validity and reliability has been criticised (Barris \& Button, 2008; Dobson \& Keogh, 2007). It is also important to note that some of the backward movement categories chosen (e.g. backward start and backward high intensity skating) were performed too infrequently by any player to be analysed. The analysis in the present study did not distinguish between movement times or frequency when the players were in control of the puck or were not and this may have influenced the performance of some of the movement activities. As well, no attempt in the current analysis was made to determine whether any differences in movement patterns were related to scoring chances, defensive success or game changing outcomes. Finally, differences in coaching strategy applied between games, periods or during game play situations were out of the control of this study but were deemed to be similar according to personal communication with the head coach. 


\subsection{Practical Applications}

The findings from this time motion, heart rate and work to rest ratio analysis of ice hockey contributes to a better understanding of how women's ice hockey is played and the physiological responses of the players during actual league games. As well, the implementation of shift times, recovery times, heart rate responses and the knowledge of work to rest ratios for shifts and the game can contribute to the development of more effective in-season and off-season conditioning programs. Furthermore, knowing the types, frequency and duration of the various movement categories may also aid in the selection of valid and reliable fitness assessments to evaluate players and monitor training; provide support in player selection; evaluate readiness to play after an injury or individualizing conditioning assignments; and, assist with designing game simulation workouts for players that are not selected to play in a league games and can experience a game-like exercise stimulus. Despite the seemingly high intensity, short duration activities and intermittent nature of ice hockey performed, the majority of the time spent and the elevated cardiovascular demand during the game would suggest that cardio-respiratory fitness is important. Recent research has shown a significant relationship between aerobic fitness and repeated shift performance in male ice hockey players (Peterson et al., 2015) that supports the current findings in female ice hockey players despite an earlier study that was unable to find a relationship between aerobic fitness and recovery from intense intermittent skating in female ice hockey players (Carey, Drake, Pliego \& Raymond, 2007). Our findings would suggest that female players require a variety of well-developed fitness qualities to perform and compete in ice hockey.

\section{Conclusions}

In summary, the novel aspect of this study was that it is the first to present the different types and time spent performing various movement patterns during competitive women's ice hockey games. The majority of female ice hockey games involved gliding on the ice and skating in the forward direction at a moderate intensity for all players. Movement activities directly related to the forward and defense positions differed as expected with the forwards performing more moderate intensity gliding and skating in the forward direction and the defense showing more backward gliding and skating during even strength play. Movement activities also varied depending on game play situations and by position. Heart rate reached near maximum levels during relatively short periods of intermittent on ice activity and recovered in between periods. Finally, shift and game work to rest ratios indicate that hockey is a high intensity, intermittent sport and these ratios varied by game play situation. This was the first study to determine the frequency and time spent performing activities specific to the game of ice hockey in female players and how these activities vary by position and game play situations unique to ice hockey.

\section{Acknowledgements}

The authors would like to thank Jessie Gill, Ben Davis, Ciaran O'Flynn, the University Hockey Team, Coaching and Training staff. This research was supported by a grant from the Sport Science Association of Alberta.

\section{References}

Barris, S. \& Button, C. (2008). A review of vision-based motion analysis in sport. Sports Medicine, 38(12), 1025-1043.

Bell, G., Game, A., Bouchard, J., Reid, C., Gervais, P., \& Snydmiller, G. (2011). Near maximal heart rate responses during a varsity ice hockey game. Applied Physiology, Nutrition and Metabolism, 36(Suppl.), S2.

Bloomfield, J., Polman, R., \& O’Donoghue, P. (2007a). Physical demands of different positions in FA premier league soccer. Journal of Sport Science and Medicine, 6(1), 63-70.

Bloomfield, J., Polman, R., O'Donoghue, P., \& McNaughton, L. (2007b). Effective speed and agility conditioning methodology for random intermittent dynamic type sports. Journal of Strength and Conditioning Research, 2(4), 10931100 .

Bracko, M. R., Fellingham, G. W., Hall, L. T., Fisher, A. G., \& Cryer, W. (1998). Performance skating characteristics of professional ice hockey forwards. Sports Medicine, Training and Rehabilitation, 8(3), 251-263.

Carey, D. G., Drake, M. M., Pliego, G. J., \& Raymond, R. L. (2007). Do hockey players need aerobic fitness? Relation between VO2max and fatigue during high-intensity intermittent skating. Journal of Strength and Conditioning Research, 21(3), 963-966.

Cox, M. H., Miles, D. S., Verde, T. J., \& Rhodes, E. C. (1995). Applied physiology of ice hockey. Sports Medicine, 19(3), 184-201.

Dillman, C. J., Stockholm, A. J., \& Greer, N. (1984). Movement of ice hockey players. International Symposium on Biomechanics in Sports, 2, 189-194.

Dobson, B. P. \& Keogh, J. W. L. (2007). Methodological issues for the application of time-motion analysis research. Strength and Conditioning Journal, 29(2), 48-55.

Falinger, C. M., Kruisselbrink, L.D., \& Fowles, J. R. (2007). Relationships to skating performance in competitive hockey players. Strength and Conditioning Journal, 21(3), 915-922. 
Fernandez-Fernandez, J., Boullosa, D.A., Sanz-Rivas, D., Abreu, L., Filaire, E., \& Mendez-Villanueva, A. (2014). Psychophysiological stress response during training and competition young female competitive tennis players. International Journal of Sports Medicine, 36(1), 22-28.

Forbes, S. C., Kennedy, M. D., \& Bell, G. J. (2013). Time-motion analysis, heart rate, and physiological characteristics of international canoe polo athletes. Journal of Strength and Conditioning Research, 27(10), 2816-2822.

Game, A., Voaklander, D., Syrotuik, D., \& Bell, G. (2003). Incidence of exercise-induced bronchospasm and exercise induced hypoxaemia in female varsity hockey players. Research in Sports Medicine, 11: 11-21.

Geithner, C. A., Lee, A. M., \& Bracko, M. R. (2006). Physical and performance differences among forwards, defensemen, and goalies in elite women's ice hockey. Journal of Strength and Conditioning Research, 20(3), 500-505.

Gilenstam, K. M., Thorsen, K., \& Henriksson-Larsen. (2011). Physiological correlates of skating performance in women's and men's ice hockey. Journal of Strength and Conditioning Research, 25(8), 2133-2142.

Green, H., Bishop, P., Houston, M., McKillop, R., Norman, R., and Stothart, P. Time-motion and physiological assessments of ice hockey performance. Journal of Applied Physiology, 40(2), 159-163.

Hockey Canada. (2014). [Online] Available: http://www.hockeycanada.ca/en-ca/Hockey-Programs/Female/StatisticsHistory.aspx (January 22, 2014).

Hopkins, W. G. (2014). [Online] Available: http://www.sportsci.org/ (June 30, 2014).

Lafontaine, D., Lamontagne, M., \& Lockwood, K. (1998). Time-motion analysis of ice-hockey skills during games. International Symposium on Biomechanics in Sport, 16, 481-484.

Linseman, M. E, Palmer, M. S, Sprenger, H. M, \& Spriet, L. L. (2014). Maintaining hydration with a carbohydrateelectrolyte solution improves performance, thermoregulation, and fatigue during an ice hockey scrimmage. Applied Physiology, Nutrition and Metabolism, 39(11), 1214-1221.

Lythe, J. \& Kilding, A. E. (2011). Physical demands and physiological responses during elite field hockey. International Journal of Sports Medicine, 32(7), 523-528.

Matthew, D., \& Delextrat, A. (2009). Heart rate, blood lactate concentration, and time-motion analysis of female basketball players during competition. Journal of Sports Sciences, 27(8), 813-821.

McInnes, S. E., Carlson, J. S., Jones, C. J., \& McKenna, M. J. (1995). The physiological load imposed on basketball players during competition. Journal of Sports Sciences, 13(5), 387-397.

Montgomery, D. L. (2006). Physiological profile of professional hockey players - a longitudinal comparison. Applied Physiology, Nutrition and Metabolism, 31(3), 181-185.

O’Donoghue, P. G. (2008). Time-motion analysis. In M. Hughes \& I. M. Franks (Eds.), The Essentials of Performance Analysis: An Introduction (pp. 180-205). New York: Routledge.

Peterson, B. J., Fitzgerald, J. S. Dietz, C. C., Ziegler, K. S., Ingraham, S. J., Baker, S. E., \& Snyder, E. M. (2015). Aerobic capacity is associated with improved repeated shift performance in hockey. Journal of Strength and Conditioning Research, 29(6), 1465-1472.

Quinney, H. A., Dewart, R., Game, A., Snydmiller, G., Warburton, D., \& Bell, G. (2008). A 26 year physiological description of a National Hockey League team. Applied Physiology, Nutrition and Metabolism, 33(4), 753-760.

Ransdell, L. B. \& Murray, T. (2011). A physical profile of elite female ice hockey players from the USA. Journal of Strength and Conditioning Research, 25(9), 2358-2363.

Rudkin, S. T. \& O’Donoghue, P. G. (2008). Time-motion analysis of first-class cricket fielding. Journal of Science and Medicine in Sport, 11(6), 604-607.

Seliger, V., Kostka, V., Grusova, D., Kovac, J., Machovcova, J., Pauer, M., \& Urbankova, R. (1972). Energy expenditure and physical fitness of ice-hockey players. European Journal of Applied Physiology, 30(4), 283-291.

Spiering, B. A., Wilson, M. H., Judelson, D. A., \& Rundell, K. W. (2003). Evaluation of cardiovascular demands of game play and practice in Women's Ice Hockey. Journal of Strength and Conditioning Research, 17(2), 329-333.

Stanula, A. \& Roczniok, R. (2014). Game intensity analysis of elite adolescent ice hockey players. Journal of Human Kinetics, 44, 211-221.

Taylor, J. (2003). Basketball: Applying time motion data to conditioning. Strength and Conditioning Journal, 25(2), $57-$ 64.

Thoden, J. S. \& Jette, M. (1975). Aerobic and anaerobic activity patterns in junior and professional hockey. Movement (Special Hockey), 2, 145-153.

Virr, J. L., Game, A., Bell, G. J. \& Syrotuik, D. (2014). Physiological Demands of women's rugby union time motion analysis and hear rate response. Journal of Sport Sciences, 32(3), 239-247. 